\title{
Pragmatic Recommendations for the Management of Anticoagulation and Venous Thrombotic Disease for Hospitalized Patients with COVID-19 in Low- and Middle-Income Countries
}

\author{
Hanan Y. Ahmed, ${ }^{1 \star}$ Alfred Papali, ${ }^{2}$ Tewodros Haile, ${ }^{1}$ Gentle S. Shrestha, ${ }^{3}$ Marcus J. Schultz, ${ }^{4,5,6}$ Ganbold Lundeg, ${ }^{7}$ and \\ Kevan M. Akrami ${ }^{8,9}$ for the COVID-LMIC Task Force and the Mahidol-Oxford Research Unit (MORU) \\ ${ }^{1}$ Division of Pulmonary and Critical Care Medicine, College of Health Sciences, Addis Ababa University, Addis Ababa, Ethiopia; ${ }^{2}$ Division of \\ Pulmonary and Critical Care Medicine, Atrium Health, Charlotte, North Carolina; ${ }^{3}$ Department of Anaesthesiology, Tribhuvan University Teaching \\ Hospital, Kathmandu, Nepal; ${ }^{4}$ Department of Intensive Care, Amsterdam University Medical Centers, Location 'Academic Medical Center', \\ Amsterdam, The Netherlands; ${ }^{5}$ Department of Clinical Tropical Medicine, Mahidol University, Bangkok, Thailand; ${ }^{6}$ Mahidol-Oxford Tropical \\ Medicine Research Unit (MORU), Mahidol University, Bangkok, Thailand; ${ }^{7}$ Critical Care and Anaesthesia Department, Mongolian National \\ University of Medical Sciences, Ulan Bator, Mongolia; ${ }^{8}$ Faculdade de Medicina, Universidade Federal da Bahia, Salvador, Brazil; ${ }^{9}$ Divisions of \\ Infectious Disease and Critical Care Medicine, University of California San Diego, San Diego, California
}

\begin{abstract}
New studies of COVID-19 are constantly updating best practices in clinical care. Often, it is impractical to apply recommendations based on high-income country investigations to resource limited settings in low- and middle-income countries (LMICs). We present a set of pragmatic recommendations for the management of anticoagulation and thrombotic disease for hospitalized patients with COVID-19 in LMICs. In the absence of contraindications, we recommend prophylactic anticoagulation with either low molecular weight heparin (LMWH) or unfractionated heparin (UFH) for all hospitalized COVID-19 patients in LMICs. If available, we recommend LMWH over UFH for venous thromboembolism (VTE) prophylaxis to minimize risk to healthcare workers. We recommend against the use of aspirin for VTE prophylaxis in hospitalized COVID-19 and non-COVID-19 patients in LMICs. Because of limited evidence, we suggest against the use of "enhanced" or "intermediate" prophylaxis in COVID-19 patients in LMICs. Based on current available evidence, we recommend against the initiation of empiric therapeutic anticoagulation without clinical suspicion for VTE. If contraindications exist to chemical prophylaxis, we recommend mechanical prophylaxis with intermittent pneumatic compression (IPC) devices or graduated compression stockings (GCS) for hospitalized COVID-19 patients in LMICs. In LMICs, we recommend initiating therapeutic anticoagulation for hospitalized COVID-19 patients, in accordance with local clinical practice guidelines, if there is high clinical suspicion for VTE, even in the absence of testing. If available, we recommend LMWH over UFH or Direct oral anticoagulants for treatment of VTE in LMICs to minimize risk to healthcare workers. In LMIC settings where continuous intravenous UFH or LMWH are unavailable or not feasible to use, we recommend fixed dose heparin, adjusted to body weight, in hospitalized COVID-19 patients with high clinical suspicion of VTE. We suggest $D$-dimer measurement, if available and affordable, at the time of admission for risk stratification, or when clinical suspicion for VTE is high. For hospitalized COVID-19 patients in LMICs, based on current available evidence, we make no recommendation on the use of serial $D$-dimer monitoring for the initiation of therapeutic anticoagulation. For hospitalized COVID-19 patients in LMICs receiving intravenous therapeutic UFH, we recommend serial monitoring of partial thromboplastin time or anti-factor Xa level, based on local laboratory capabilities. For hospitalized COVID-19 patients in LMICs receiving LMWH, we suggest against serial monitoring of anti-factor Xa level. We suggest serial monitoring of platelet counts in patients receiving therapeutic anticoagulation for VTE, to assess risk of bleeding or development of heparin induced thrombocytopenia.
\end{abstract}

\section{INTRODUCTION}

Current recommendations on anticoagulation for hospitalized patients with COVID-19 is based mainly on evidence from high-income countries (HICs). It may not be practical to apply these recommendations to resource-limited settings, particularly in low- and middle-income countries (LMICs). Indeed, high dependency units and intensive care units (ICUs) in LMICs are frequently restricted in the availability of infrastructure, equipment, medications, skilled nurses, and clinicians. An international task force comprised members from LMICs and HICs, all with direct experience in various LMIC settings, critically appraised a list of questions regarding anticoagulation and thrombotic disease for hospitalized patients with COVID-19. Herein, we provide a list of recommendations and suggestions after pragmatic, experience-based appraisal of the literature. The recommendations are summarized in Table 1.

\footnotetext{
* Address correspondence to Hanan Y. Ahmed, Division of Pulmonary and Critical Care Medicine, College of Health Sciences, Addis Ababa University, Gambia St., Addis Ababa 2380, Ethiopia. E-mail: hananyusufahmed@gmail.com
}

\section{METHODS}

A full description of the methods is provided in the Appendix. Briefly, we formulated a set of questions regarding anticoagulation for patients with COVID-19. The list was reviewed for content and clarity by other members of the COVID-LMIC Task Force. After approval, the anticoagulation subgroup assigned one or two members to search the literature for evidence to answer each of the questions. The literature search was performed in a minimum of one general database (e.g., MEDLINE and EMBASE) and the Cochrane Libraries, including articles pertaining to COVID-19, SARS, middle east respiratory syndrome (MERS), and other respiratory viruses. We selected relevant publications, appraised the evidence and classified the quality of evidence as high, moderate, low, or very low. Recommendations were rated as strong or weak, depending on the quality of evidence and several other factors, such as availability, affordability, and feasibility in LMICs. A strong recommendation was worded as "we recommend ..." and a weak recommendation as "we suggest ...," followed by the quality of evidence. A number of recommendations could remain "ungraded" (UG), when, in the opinion of the subgroup members, such recommendations were not conducive for the process aforementioned (Appendix Table 2). 
TABLE 1

Recommendations and suggestions for the management of anticoagulation and thrombotic disease for hospitalized patients with COVID-19 in lowand middle-income countries (with grading)

1. Prophylaxis

In the absence of contraindications, we recommend prophylactic anticoagulation with either LMWH or unfractionated heparin (UFH) for all hospitalized COVID-19 patients in LMICs (strong recommendation and moderate quality of evidence)

If available, we recommend LMWH over UFH for VTE prophylaxis to minimize risk to healthcare workers (UG best practice statement)

We recommend against the use of aspirin for VTE prophylaxis in hospitalized COVID-19 and non-COVID-19 patients in LMICs (strong recommendation and low quality of evidence)

Because of limited evidence, we suggest against the use of "enhanced" or "intermediate" prophylaxis in COVID-19 patients in LMICs. (weak recommendation and low quality of evidence)

In hospitalized COVID-19 patients in LMICs, based on current available evidence, we recommend against the initiation of empiric therapeutic anticoagulation without clinical suspicion for VTE (strong recommendation and low quality of evidence)

If contraindications exist to chemical prophylaxis, we recommend mechanical prophylaxis with intermittent pneumatic compression devices or graduated compression stockings for hospitalized COVID-19 patients in LMICs (strong recommendation and low quality of evidence)

2. Management In LMICs, we recommend initiating therapeutic anticoagulation for hospitalized COVID-19 patients, in accordance with local clinical practice guidelines, if there is high clinical suspicion for VTE, even in the absence of testing (strong recommendation and low quality of evidence)

If available, we recommend LMWH over UFH or direct oral anticoagulants for treatment of VTE in LMICs to minimize risk to healthcare workers (UG best practice statement)

In LMIC settings where continuous intravenous UFH or LMWH are unavailable or not feasible to use, we recommend fixed dose heparin, adjusted to body weight, in hospitalized COVID-19 patients with high clinical suspicion of VTE (weak recommendation and low quality of evidence)

3. Monitoring For hospitalized COVID-19 patients in LMICs we suggest $D$-dimer measurement, if available and affordable, at the time of admission for risk stratification, or when clinical suspicion for VTE is high (weak recommendation and low quality of evidence)

For hospitalized COVID-19 patients in LMICs, based on current available evidence, we make no recommendation on the use of serial $D$-dimer monitoring for the initiation of therapeutic anticoagulation

For hospitalized COVID-19 patients in LMICs receiving intravenous therapeutic UFH, we recommend serial monitoring of partial thromboplastin time or anti-factor Xa level, based on local laboratory capabilities (strong recommendation and high quality of evidence)

For hospitalized COVID-19 patients in LMICs receiving LMWH, we suggest against serial monitoring of anti-factor Xa level (weak recommendation and low quality of evidence)

For hospitalized COVID-19 patients in LMICs, we suggest serial monitoring of platelet in patients receiving therapeutic anticoagulation for VTE, to assess risk of bleeding or development of HIT (weak recommendation and low quality of evidence)

LMWP = low molecular weight heparin; UFH = unfractionated heparin; VTE = venous thromboembolism; UG = ungraded.

The recommendations were reviewed by the anticoagulation subgroup in an iterative process and were later reviewed by the entire task force in three rounds. This document represents a rapid clinical review, rather than an exhaustive metaanalysis, that aims to offer practical treatment approaches in LMICs based on the best available evidence.

\section{QUESTIONS}

Three relevant and clearly defined questions regarding COVID-19 and thrombosis were formulated.

1. What type of venous thromboembolism (VTE) prophylaxis should hospitalized patients with COVID-19 receive in LMICs?

2. How should clinically suspected or confirmed COVID-19related VTE be managed in LMICs?

3. How should COVID-19 patients on treatment for VTE be routinely monitored in LMICs?

What type of VTE prophylaxis should hospitalized patients with COVID-19 receive in LMICs? Rationale. COVID19 appears to predispose patients to thrombotic disease, both in venous and arterial circulations, due to excessive inflammation, platelet activation, endothelial dysfunction, and stasis. ${ }^{1}$ Prophylactic anticoagulation, although most effective in surgical and orthopedics patients, is known to reduce the risk of VTE in acutely ill hospitalized medical patients. ${ }^{2}$ Patients hospitalized for COVID-19, especially those requiring intensive care, are increasingly recognized to be at higher risk for VTE. ${ }^{3}$ The WHO, ${ }^{4}$ the American $\mathrm{NIH},{ }^{5}$ and International Society on Thrombosis and Haemostasis, ${ }^{6}$ among other societies, ${ }^{7,8}$ recognize that all hospitalized COVID-19 patients are at high risk for VTE. Therefore, the standard of care is to provide prophylactic anticoagulation for all hospitalized COVID-19 patients irrespective of other traditional risks for VTE. With this understanding, we compiled the evidence to date regarding VTE prophylaxis to generate recommendations best suited for LMICs.

Search results. A PubMed search was performed to identify articles as of June 24, 2020, using the following search and/or Medical Subject Headings (MeSH) terms: "COVID-19," OR "SARS-CoV-2," OR "2019-nCOV," OR "Novel Coronavirus" AND "prophylaxis," OR "Anticoagulant therapy," OR "pneumatic compression." Six studies from HICs and one from China directly answering the question and a total of 14 other relevant studies to the question were initially identified. During the revision process, an updated literature search was performed through November 22, 2020. Ten additional relevant articles, including three systematic reviews and one survey on anticoagulation practice in COVID-19 patients, were evaluated.

Evidence. Hospitalized COVID-19 patients have demonstrated greater risk for the development of VTE. A Dutch study 
evaluating the incidence and composite outcome of VTE and arterial thrombotic complications in 184 COVID-19 patients found a $31 \%$ incident rate of thrombotic events. Pulmonary embolism (PE) was the most frequent thrombotic complication, contributing to $81 \%$ of the events. ${ }^{9}$ Survival among patients with incident and recurrent VTE is significantly reduced, especially after PE. ${ }^{10,11}$ The combination of COVID-19 infection and resultant inflammation may underlie the autopsy findings of small-vessel hyaline thrombus formation. ${ }^{12}$ In addition, COVID-19 patients have many VTE risk factors including, but not limited to, dehydration with poor appetite and gastrointestinal manifestations, immobility, obesity, and other concomitant comorbidities. $^{13}$

In summary, the risk of VTE in patients hospitalized with COVID-19 is significant and all patients, barring contraindications, are eligible for prophylaxis. Various risk assessment models (RAMs) are available to help risk stratify development of VTE; however, most RAMs should qualify COVID-19 patients for prophylaxis because of severity of their illness. ${ }^{14}$

Standard chemoprophylaxis. With respect to how VTE prophylaxis is achieved, heparin products, in addition to their well-known anticoagulant properties, appear to have additional antiviral and anti-inflammatory effects that may be potentially beneficial in hospitalized COVID-19 patients. ${ }^{15} \mathrm{~A}$ Cochrane database systematic review of heparin products for VTE prevention in non-COVID-19 patients revealed six trials comparing low molecular weight heparin (LMWH) with unfractionated heparin (UFH) for medical patients. Compared with UFH, LMWH reduced the risk of deep vein thrombosis (DVT) (OR: $0.77 ; 95 \% \mathrm{Cl}: 0.62-0.96 ; P=0.02$ ) and major bleeding (OR: $0.43 ; 95 \% \mathrm{Cl}: 0.22-0.83 ; P=0.01$ ). There was no clear evidence that the effects of LMWH and UFH differed for PE outcomes, all-cause mortality, and thrombocytopenia. ${ }^{16}$

Mechanical prophylaxis. For patients where use of heparin products is contraindicated, mechanical prevention with intermittent pneumatic compression (IPC) devices or graduated compression stockings (GCS) are non-pharmacologic options for VTE prophylaxis. A systematic review, before the era of COVID-19, which included 70 trials (two from Turkey and two from China both upper middle income countries) involving 16,164 patients, found that IPC reduced VTE occurrence compared with no IPC prophylaxis, but with a reduced risk of bleeding. The same review demonstrated that addition of pharmacological prophylaxis to IPC further reduced the risk of DVT compared with IPC alone, emphasizing the importance of transitioning to pharmacologic prophylaxis as soon as the bleeding risk is reduced. ${ }^{17}$

However, the opposite approach-adding mechanical prophylaxis to pharmacological prophylaxis-was not found to be beneficial in a recent randomized control trial involving non-COVID-19 patients. ${ }^{18}$ We were unable to identify any study from either HICs or LMICs that reported on use of mechanical prophylaxis in COVID-19 patients. Reports around the world also show that mechanical thromboprophylaxis is not widely used. ${ }^{19}$

Although aspirin may be cheap and widely available in LMIC settings, the safety and efficacy of aspirin for VTE prophylaxis remains unknown. In those with cardiovascular disease, a recent cohort study of 412 COVID-19 patients assessed the use of aspirin within 24 hours of hospital admission or in the 7 days before hospital admission found that use was independently associated with a lower risk of mechanical ventilation, ICU admission, and in-hospital mortality. Although a reduction in microthrombosis is a plausible mechanism, the study did not observe a lower rate of overt thrombosis in aspirin users. ${ }^{20}$ The benefit of aspirin beyond the cardiovascular indications in COVID-19 patients remains to be seen.

"Enhanced" chemoprophylaxis. The clinical benefit of "enhanced," "intermediate," or "high-intensity" chemoprophylaxis using a higher dose (often twice the standard prophylaxis dose), although less than therapeutic levels, remains controversial. ${ }^{21}$ Several observational studies from the United States, ${ }^{22}$ The Netherlands, ${ }^{9}$ France, ${ }^{23}$ and China $^{24}$ suggest that routine prophylactic dose anticoagulation might be insufficient to prevent the occurrence of VTE in certain high-risk COVID-19 patients, such as those with coagulation abnormalities by routine laboratory analysis of $D$-dimer, international normalized ratio (INR), and partial thromboplastin time (PTT) among others. This may be reflected particularly in those admitted to the ICU, where the reported incidence of primarily venous thrombotic complications ranges from $31 \%$ to $69 \%$ among COVID-19 patients. ${ }^{3,24,25}$

A small retrospective study of 49 mechanically ventilated patients from Belgium reported that the use of high-intensity thromboprophylaxis was associated with a lower occurrence of PE. ${ }^{26}$ Similarly, a Swedish study of 152 patients demonstrated reduced incidence of thromboembolic events and mortality in those receiving high-dose chemoprophylaxis versus "medium" or "low" (high $13.5 \%$ versus medium $25 \%$ versus low $38.8 \% ; P=0.02$ ). Importantly, there was no difference in the development of bleeding events among the three groups. ${ }^{27}$ Similar findings were identified in a retrospective study from Abu Dhabi albeit finding that therapeutic anticoagulation, rather than enhanced chemoprophylaxis, was associated with major bleeding. ${ }^{28}$ Another retrospective observational study of 468 hospitalized patients in the United States found that initial use of enhanced dosing thromboprophylaxis correlated with a downtrend in $D$-dimer levels and was associated with improved 30 -day mortality without a significant increased rate of bleeding. ${ }^{29}$ In contrast to these studies, a prospective Italian study involving 74 patients found no difference in the incidence of VTE events in those with either enhanced or therapeutic dosed anticoagulation compared with standard dosing. Six patients in this study developed severe hemorrhagic complications, and VTE events were still diagnosed despite intermediate or therapeutic dose of anticoagulation. ${ }^{30}$

Therapeutic dosing as VTE prophylaxis. Although prophylaxis against VTE for hospitalized COVID-19 patients is established as standard of care, it remains unclear if treatment dose anticoagulation improves outcomes without increasing bleeding risk in those without a clinical suspicion for VTE. Studies to date of varying quality and design have yielded contradictory results. A large retrospective cohort study of 2,773 hospitalized COVID-19 patients from the United States found no difference in hospital mortality in those with and without treatment dose anticoagulation (22.5\% versus $22.8 \%){ }^{31}$ Two other retrospective studies similarly from the United States found no survival difference for COVID-19 patients treated with therapeutic anticoagulation compared with those on prophylactic dosing after propensity score-matching. ${ }^{32,33}$ Similarly, a study involving 4,389 patients from five New York City hospitals identified no significant mortality difference between prophylactic and therapeutic dosed patients $(P=0.08)$, although not surprisingly found lower 
in-hospital mortality in those with therapeutic and prophylactic dosed anticoagulation than those without prophylaxis. ${ }^{34}$

Another retrospective multicenter cohort study from the United States that included 3,480 patients demonstrated that anticoagulation was associated with reduced risk of death both at prophylactic and therapeutic doses compared with no prophylaxis. Although enhanced prophylaxis was associated with prolonged survival, especially in critically ill patients, excess non-disabling bleeding was witnessed in this group. ${ }^{35}$

These studies, among others, form the evidence base for two systematic reviews and meta-analysis assessing the association of VTE with different anticoagulation doses in hospitalized COVID-19 patients. One review of 16 studies found that both therapeutic and prophylactic dosing were associated with lower risk of mortality. ${ }^{36}$ The other review included 25 observational studies finding that therapeutic anticoagulation was not associated with a lower mortality risk. ${ }^{37}$

Other studies sought to differentiate whether a subset of patients may benefit from therapeutic anticoagulation for VTE prophylaxis compared with standard dosing. A retrospective secondary analysis of 141 intubated COVID-19 patients from three hospitals in the United States found no difference in 28day mortality from time of intubation when empirically treated with therapeutic dose anticoagulation compared with standard DVT prophylactic doses, even among those with $D$-dimer levels $>2 \mu \mathrm{g} / \mathrm{mL} .{ }^{38}$ As only intubated patients were included, it is unclear whether therapeutic anticoagulation in a general medical ward may reduce progression to intubation or incidence of VTE. A retrospective study from the United States analyzed 3,625 COVID-19 patients with moderate or severe illness finding that therapeutic anticoagulation including apixaban has similar efficacy to enoxaparin in decreasing mortality in hospitalized COVID-19 patients. $^{39}$

Based on these findings, it remains unclear whether therapeutic anticoagulation offers a mortality benefit in critically ill patients with or without intubation, or stratified by $D$-dimer. Furthermore, clinically significant adverse bleeding events are higher in those receiving therapeutic anticoagulation than those on standard chemoprophylaxis. Rigorous prospective studies are required to further evaluate the role of therapeutic dosing for prophylaxis. It may be that the primary driver of mortality in these patients is the degree of severity of infection rather than initiation of therapeutic anticoagulation. ${ }^{40}$

Availability, feasibility, affordability, and safety. Guidelines for chemoprophylaxis for non-COVID-19 cases in LMICs frequently are in accordance with recommendations from HICs. ${ }^{41}$ Although many strategies including various RAMs have been proposed to improve the practice of VTE prophylaxis and treatment, adherence remains suboptimal. ${ }^{42-44}$

Most LMIC-based studies on adherence to VTE prophylaxis, before the era of COVID-19, report a range of $30-60 \%$, despite a high percentage of patients at risk. ${ }^{45,46}$ Among the explanations for this relative underutilization of thromboprophylaxis described was lack of physicians' knowledge. ${ }^{47}$ Other studies, including those from HICs, have cited lack of awareness of both the disease and evidencebased guidelines. ${ }^{48,49}$ Many practitioners seem to believe that VTE is an uncommon diagnosis (based on their own clinical experiences) and therefore VTE prevention may not be considered necessary. ${ }^{50}$ This thinking is contrary to findings from several studies demonstrating that Asians have significant risk factors for and increasing incidence of VTE. ${ }^{51}$
A recent global survey involving 515 physicians from 41 countries, of which $<10 \%$ were from LMICs, assessed current physician anticoagulation practice for COVID-19 patients. The majority of respondents (78\%) recommended prophylactic anticoagulation for all hospitalized patients with COVID-19, whereas $43 \%$ would follow institutional guidelines for criteria for prophylaxis. Two-thirds recommended LMWH as the drug of choice, whereas the remaining recommended UFH. ${ }^{52}$ Whereas standard prophylactic dosing for VTE appears well tolerated in both non-COVID-19 and COVID-19 hospitalized patients, the safety of enhanced and therapeutic dosing for VTE prophylaxis, especially in those without clinical suspicion for VTE, remains controversial given a significant risk of bleeding. $^{29,53,54}$

Bleeding complications in hospitalized COVID-19 patients may be higher than expected. An observational study from the United States identified a trend toward higher incidence of bleeding events in fully anticoagulated, intubated COVID-19 patients than their non-intubated counterparts (intubated 30/ $395 ; 7.5 \%$ versus non-intubated $32 / 2,378 ; 1.35 \%) .{ }^{31}$ Another study from the United States found that $19(0.5 \%)$ patients developed hemorrhagic stroke, in which therapeutic anticoagulation was prescribed in $89.5 \%$ of these patients. ${ }^{55}$

Full anticoagulation is now being provided for hospitalized COVID-19 patients in many centers. However, rates of bleeding in such patients appear to be high. Therefore, based on available evidence, we do not endorse routine full anticoagulation for hospitalized patients with COVID-19. Rigorous prospective studies to better understand the risk-benefit of therapeutic or enhanced dose anticoagulation in the absence of documented or clinical suspicion of VTE is needed. ${ }^{56}$

Pharmacological prophylaxis is generally available in LMICs and can be delivered feasibly and safely. The WHO Essential Medicines List includes both heparin and enoxaparin. ${ }^{57} \mathrm{Me}-$ chanical modalities may not be available in many LMIC settings and there is the additional challenge of ensuring proper use, although mechanical prophylaxis has the advantage of reusability.

The higher drug cost of LMWH has been mentioned as a reason for using UFH for VTE prevention before the era of COVID-19. ${ }^{58}$ A review from Latin America supports the preferred use of treatment-dose heparin over LMWH. ${ }^{59}$ One hospital-based study performed in a HIC involving acute ischemic stroke patients determined that the average cost per patient due to VTE or bleeding events was lower with prophylactic enoxaparin versus UFH (US $\$ 422$ versus US $\$ 662$, respectively; net savings US\$240). The average anticoagulant cost, including drug administration cost per patient, was lower with UFH versus enoxaparin (US\$259 versus US\$360, respectively; net savings US\$101). However, when both clinical events and drug acquisition costs were considered, the total hospital cost was lower with enoxaparin versus UFH (US $\$ 782$ versus US\$922, respectively; savings US\$140). ${ }^{60}$ A similar conclusion was reached from a different U. S.-based study, which found total average costs per patient of US\$1,264 for enoxaparin, US\$1,585 for UFH, and US $\$ 2,245$ for no prophylaxis. It was unclear why enoxaparin prophylaxis was more costly than UFH prophylaxis. For the healthcare payer, considering all direct medical costs associated with VTE up to 2 years after an admission for acute illness, prophylaxis with enoxaparin appears to be more effective and less costly than UFH. ${ }^{61}$ Similar comprehensive studies have not been 
performed in LMIC settings. Estimation of total cost for pharmacologic VTE prophylaxis, rather than only direct drug cost, might aid in medication choice for policy makers in LMICs.

In the context of COVID-19, another consideration is the frequency of dosing. Unfractionated heparin prophylaxis is often administered twice or thrice daily, whereas LMWH, especially enoxaparin, is dosed daily. International guidelines also state that once-daily regimens of LMWH may be advantageous over UFH because missed doses may be associated with worse outcomes, will reduce healthcare worker exposure, and conserve personal protective equipment. ${ }^{13,62}$

Recommendations and suggestions (Table 1).

1) In the absence of contraindications, we recommend prophylactic anticoagulation with either LMWH or UFH for all hospitalized COVID-19 patients in LMICs (strong recommendation and moderate quality of evidence).

2) If available, we recommend LMWH over UFH for VTE prophylaxis to minimize risk to healthcare workers (UG best practice statement).

3) We recommend against the use of aspirin for VTE prophylaxis in hospitalized COVID-19 and non-COVID-19 patients in LMICs (strong recommendation and low quality of evidence).

4) Because of limited evidence, we suggest against the use of "enhanced" or "intermediate" prophylaxis in COVID-19 patients in LMICs (weak recommendation and low quality of evidence).

5) In hospitalized COVID-19 patients in LMICs, based on current available evidence, we recommend against the initiation of empiric therapeutic anticoagulation without clinical suspicion for VTE (strong recommendation and low quality of evidence).

6) If contraindications exist to chemical prophylaxis, we recommend mechanical prophylaxis with IPC devices or GCS for hospitalized COVID-19 patients in LMICs (strong recommendation and low quality of evidence).

How should clinically suspected or confirmed COVID19-related VTE be managed in LMICs? Rationale. Management of VTE requires timely diagnosis, knowledge of risk factors for both thrombosis and bleeding, safe administration of medications, and ability to monitor, when indicated. Clinical suspicion for PE should arise if a patient develops a sudden deterioration of oxygenation, respiratory distress, or hypotension disproportional to pulmonary involvement by COVID19. According to COVID-19 clinical management guidelines from the $\mathrm{WHO},{ }^{4} \mathrm{NIH},{ }^{5}$ and various societies, ${ }^{6,7}$ patients with suspected or confirmed VTE should receive therapeutic anticoagulation as would be indicated in those without COVID-19. The optimal approach to managing VTE related to COVID-19 in LMICs remains unknown.

Search results. MEDLINE, Embase, and Web of Science were searched through the end of May 2020 using combinations of $\mathrm{MeSH}$ terms and free text words, including "COVID-19" OR "SARS-COV-2" OR "2019-nCOV" OR "Novel Coronavirus" AND "critically ill” AND "Anticoagulant therapy." Fourteen studies were initially included to answer the question. Except those reported from China, no studies from LMICs were found directly answering the question. During the revision process, an updated literature search was performed through November 22, adding 16 relevant studies, two meta- analyses, and one global survey regarding use of anticoagulation during COVID-19 from 41 different countries, including LMICs.

Evidence. Heparins (parenteral and subcutaneous). A large, retrospective study from New York (United States) suggested that empiric therapeutic anticoagulation with heparin (mainly LMWH) may have a mortality advantage among hospitalized patients with COVID-19 and suspected VTE. ${ }^{31}$ Subsequent studies found improved outcomes with therapeutic anticoagulation with heparin as the primary agent. ${ }^{34}$ A comparative review of societal recommendations found that $L M W H$ is preferred over UFH by Anticoagulation Forum and American College of Chest Physicians, whereas LMWH and UFH are recommended as first line by CDC and American Society of Hematology. All recommendations acknowledge the inherent variations of the different heparin products and necessary changes depending on comorbidities (i.e., renal or hepatic dysfunction, gastrointestinal function, and thrombocytopenia).

Parenteral anticoagulation (i.e., UFH) may be preferred in critically ill patients that may require invasive procedures as it may be held temporarily and has no known drug-drug interactions with COVID-19 therapies. Low molecular weight heparin may be preferred in patients who are unlikely to need procedures given concerns with UFH regarding the time to achieve therapeutic PTT and increased exposure to healthcare workers. ${ }^{62}$ Heparins are also thought to have effects other than anticoagulation in COVID-19 patients. It is thought that heparins bind to COVID-19 spike proteins and downregulate interleukin-6 (IL-6) ${ }^{63}$ the levels of which have been shown to be elevated in COVID-19 patients. ${ }^{64}$

Direct oral anticoagulants (DOACs). Direct oral anticoagulant use during inpatient management has been reported in recent studies. Benefits of DOACs include lack of need for laboratory monitoring, facilitation of discharge planning and outpatient management. Direct oral anticoagulants, however, have a longer half-life than UFH and LMWH, which may complicate urgent invasive procedures and development of renal impairment. ${ }^{65}$ Another risk may include a potential bioavailability-related effect on clinical effectiveness through interactions with drugs including dexamethasone. Dexamethasone can decrease DOAC levels through strong P-gp and CYP3A4 induction. ${ }^{13}$

A study enrolling 12 consecutive patients on DOACs who were hospitalized with COVID-19 showed that for each patient, pre-dose trough concentration ( $C$-trough) DOAC levels were 6 times higher during hospitalization than in the prehospitalization period. ${ }^{66}$ The finding may be attributed to gastrointestinal and renal dysfunction, which are common problems in hospitalized severe COVID-19 patients.

In a prospective Italian study of 844 COVID-19 patients, $n=$ $65(7.7 \%)$ were taking DOACs before hospitalization. Patients on DOAC developed acute hypoxemic respiratory failure more frequently than non-DOAC patients and experienced a higher mortality rate $(44.6 \%$ versus $19.8 \%, P<0.001) .{ }^{67}$

Once there is clinical improvement and plan to discharge, patients with VTE can be switched to a DOAC or vitamin $\mathrm{K}$ antagonist with plan to treat for at least a total of 3 months.

Thrombolytics. International societies recommend thrombolytics in patients with objectively confirmed PE with hemodynamic instability or signs of obstructive shock who are not at high risk of bleeding. Peripheral thrombolysis is recommended over catheter-directed thrombolysis. ${ }^{8}$ Studies 
reporting on systemic thrombolysis in COVID-19 patients are limited to small case series. ${ }^{68}$

Availability, feasibility, affordability, and safety. Frequent inpatient monitoring of serum coagulation parameters may be feasible for some LMIC facilities, but limited in many others. In situations where the costs of inpatient laboratory testing are passed to the patient or the family, these costs can quickly become prohibitive, so laboratory testing must be ordered judiciously.

The most common anticoagulant used in many LMIC settings for initial inpatient treatment is UFH. The major feasibility issue with UFH is the need for frequent blood draws to monitor activated partial thromboplastin time (APTT) levels. ${ }^{69,70}$

Limited laboratory capacity in some LMIC settings may preclude safe inpatient administration of continuous UFH infusion, although a fixed-dose weight and renal adjusted UFH protocol may be a safe alternative where LMWH is unavailable. Low molecular weight heparin requires no laboratory monitoring, but is typically more expensive for inpatients and may not be as readily available in some settings. Despite the high drug cost, studies from Europe and Brazil suggest that outpatient treatment of VTE with LMWH may save as much as US $\$ 1,641$ per patient compared with inpatient UFH therapy. ${ }^{71,72}$ This cost saving analysis likely is irrelevant for the hospitalized COVID-19 patient given the low likelihood of outpatient therapy for this disease.

After the initial heparin-based therapy, the most common outpatient anticoagulant used in LMICs is warfarin. Warfarin is well studied, having been used for many years, and low cost generic versions are readily available throughout the world. ${ }^{73}$ The need for and costs associated with necessary laboratory monitoring of INR for warfarin patients, however, may be difficult in most resource-limited settings in LMICs. Even in facilities with relatively ample resources, appropriate treatment based on INR can be challenging. ${ }^{74}$

Recommendations and suggestions (Table 1).

1) In LMICs, we recommend initiating therapeutic anticoagulation for hospitalized COVID-19 patients with high clinical suspicion of VTE, in accordance with local clinical practice guidelines, even in the absence of testing (strong recommendation and low quality of evidence).

2) If available, we recommend LMWH over UFH or DOACs for treatment of VTE in LMICs to minimize risk to healthcare workers (UG best practice statement).

3) In LMIC settings where continuous intravenous UFH or LMWH are unavailable or not feasible, we recommend fixed dose heparin, adjusted to body weight and renal function, in hospitalized COVID-19 patients with high clinical suspicion of VTE (weak recommendation and low quality of evidence)

How should COVID-19 patients on treatment for VTE be routinely monitored in LMICs? Rationale. In COVID-19 patients, monitoring of parameters associated with coagulation (including among others INR, PTT, and $D$-Dimer) is a dynamic and ongoing process. Before the initiation of VTE treatment, abnormal coagulation parameters have been shown to be more common in non-survivors than survivors. ${ }^{52}$ Although patients are undergoing treatment for VTE, monitoring is required to assess efficacy and emergence of possible complications.

Patients hospitalized with COVID-19 may have comorbidities necessitating other medications with the potential for drug-drug interaction. These patients may rapidly develop severe disease with complications such as renal failure, respiratory failure, or liver dysfunction that can affect both VTE and bleeding risk.

Search results. A PubMed search was performed to identify articles as of June 24, 2020, using the search terms "VTE," "monitoring," "laboratory," "COVID-19," "SARS-CoV-2," and "antithrombotic therapy." The search resulted in five retrospective cohort studies directly answering the question, all of which were from HICs. In addition, there were two pertinent studies and one registry from LMICs and several relevant studies from HICs, including a Cochrane meta-analysis. During the revision process, an updated literature search was performed through November 22, adding five more relevant studies.

Evidence. Although bedside examination for signs/symptoms of bleeding or hemorrhage is important, the fundamental approach to monitoring patients on therapeutic anticoagulation with or without COVID-19 is based on laboratory parameters. In a Chinese observational study of 407 patients identified as high risk of VTE according to the Padua Score, 44 (11\%) also had a high risk of bleeding by the IMPROVE bleeding score. For these patients, the dose and duration of anticoagulants needed to be adjusted. ${ }^{75}$

In COVID-19 patients, the evidence to support laboratory monitoring for coagulopathy is limited. Elevated levels of blood IL-6, high-sensitivity cardiac troponin I, and lactate dehydrogenase, as well as lymphopenia and thrombocytopenia, are more variably reported in severe COVID-19. ${ }^{76-78}$

$D$-dimer. There is emerging evidence for $D$-dimer measurement. A retrospective, single-center, observational study from China involving 183 hospitalized patients with COVID-19 pneumonia demonstrated significantly higher $D$-dimer level at admission in non-survivors than in survivors $(2.12 \mu \mathrm{g} / \mathrm{mL}$ versus $0.61 \mu \mathrm{g} / \mathrm{mL} ; P<0.001$; normal $D$-dimer level $<0.50$ $\mu \mathrm{g} / \mathrm{mL}$ ). Moreover, non-survivors had evidence of progressive disseminated intravascular coagulation, with decreased fibrinogen, increased $D$-dimer, and increased prothrombin time (PT), 10 days after admission. ${ }^{11}$ Although evidence at the time of writing remains limited, other studies also have suggested clinical utility of $D$-dimer measurement. A retrospective French study involving 71 hospitalized (non-ICU) COVID-19 patients reported a $90 \%$ negative predictive value of baseline $D$-dimer level $<1.0 \mu \mathrm{g} / \mathrm{mL}$ for VTE and $98 \%$ for PE. ${ }^{79}$ The same study reported a positive predictive value for VTE of $44 \%$ and $67 \%$ for PE with $D$-dimer levels $\geq 1.0 \mu \mathrm{g} / \mathrm{mL}$ and $\geq 3 \mu \mathrm{g} / \mathrm{mL}$, respectively.

Other studies have identified laboratory based risk stratification models using $D$-dimer thresholds to identify patients who should receive prophylactic or therapeutic anticoagulation, even with low clinical suspicion of VTE. ${ }^{80}$ Those with $D$-dimer level persistently $<1,000 \mu \mathrm{g} / \mathrm{L}$ would receive standard prophylactic dosing, whereas those with initial level $<1,000 \mu \mathrm{g} / \mathrm{L}$ on admission, but with a significant increase during hospitalization to levels above 2,000-4,000 $\mu \mathrm{g} / \mathrm{L}$, may consider imaging for DVT or PE, particularly if the patient is symptomatic for these conditions. When imaging is not feasible, and clinical suspicion for VTE is high, therapeutic LMWH without imaging is recommended, provided that the risk of bleeding is acceptable. In a multicenter study involving three U.S. academic hospitals ${ }^{22}$ elevated $D$-dimer at hospital presentation and an elevated $D$-dimer or decreased platelet count during ICU admission suggested development of VTE, despite prophylactic anticoagulation. A serum $D$-dimer concentration greater than 2,600 ng/mL (normal range, 0-292 ng/mL) demonstrated the most accurate discriminatory function in this study to detect the occurrence of VTE (area under the curve 
[AUC]: $0.760 ; P<0.0001$; sensitivity and specificity of $89.7 \%$ and $59.5 \%$, respectively).

The utility of $D$-Dimer measurement in hospitalized patients with COVID-19 has been explored in multiple recent studies. A retrospective observational study of 114 patients from the United States found that during the first 7 days of hospitalization, peak $D$-dimer level of $>2,500 \mathrm{ng} / \mathrm{mL}$ and a rate of change exceeding $150 \mathrm{ng} / \mathrm{mL} /$ day were predictive of future diagnosis of VTE, but not mortality. ${ }^{81}$ A study from England with 974 patients found a $D$-dimer cutoff value of $2,247 \mathrm{mcg} / \mathrm{L}$ correlates with a reasonable discriminate function (sensitivity of 0.72 and specificity of 0.74 ) in the diagnosis of VTE in patients with moderate-to-severe COVID-19 disease. ${ }^{82} \mathrm{~A}$ French study reported a better predictive performance of $D$ dimer cutoff value of $2,590 \mathrm{ng} \cdot \mathrm{mL}^{-1}$ (AUC: $0.88, P<0.001$, sensitivity $83.3 \%$, specificity $83.8 \%$ ) among those confirmed to have PE. The authors further specified that values above $2,590 \mathrm{ng} \cdot \mathrm{mL}^{-1}$ were associated with a 17 -fold increase in the adjusted risk of PE. ${ }^{83}$ Based on these studies, elevated $D$ dimers levels in those with clinical suspicion of VTE may aid in diagnosis of PE in hospitalized COVID-19 patients and support the wider use of $D$-dimer guided screening for $P E$ in acutely ill hospitalized patients with COVID-19.

Prothrombin time (PT), partial thromboplastin time (PTT), and factor $\mathrm{Xa}$. Subcutaneous LMWH and fixed dosed heparin require laboratory monitoring. For patients on therapeutic UFH, serial monitoring of (PTT; also known as APTT) or factor $\mathrm{Xa}$ is suggested. The benefit of monitoring IV heparin once a therapeutic threshold has been exceeded is not well defined. ${ }^{84}$

Anticoagulation Forum and American College of Chest Physicians both recommend monitoring anti-Xa levels to monitor UFH because of baseline artefactual abnormalities in PTT and a potential for heparin resistance. None of the guidelines recommend dosing of LMWHs based on anti-Xa levels given lack of evidence on outcomes for thrombosis or bleeding. Body weight-adjusted doses for LMWH appear safer, which may be particularly effective in overweight patients. Elevated PT or PTT is not a contraindication to thromboprophylaxis in the absence of active bleeding. A platelet count $<25 \times 10^{9} / \mathrm{L}$ or fibrinogen $<0.5 \mathrm{~g} / \mathrm{L}$ constitutes a bleeding risk, warranting holding of standard dose VTE prophylaxis, whereas therapeutic anticoagulation should be withheld if platelet count $<50 \times 10^{9} / \mathrm{L}$ or fibrinogen $<1.0 \mathrm{~g} / \mathrm{L}$. ${ }^{62}$

The role of serial hemoglobin monitoring in COVID-19 patients on therapeutic anticoagulation is poorly defined.

We found no data from LMICs regarding the diagnosis of heparin induced thrombocytopenia (HIT). Speculative explanations for this finding include limited availability of laboratory assays for serological confirmation of HIT, expense associated with using nonheparin anticoagulants, and a lack of awareness of the guidelines.

Availability, feasibility, affordability, and safety. A study from Nigeria identified the inability to monitor as a primary reason for reduced clinician adherence to anticoagulation guidelines. ${ }^{85}$ In a separate multicenter survey from six southeastern Nigerian tertiary hospitals, most respondents were able to recognize PT and APTT, but not anti-Xa assay, as laboratory monitoring tools for anticoagulation therapy. This finding correlated with heparin and warfarin as the most commonly administered anticoagulants among respondents. ${ }^{86}$

$D$-dimer testing might not be readily available in some LMICs and likely would incur a higher cost, although specific pricing in various LMIC environments remains unclear. Early COVID-19 studies suggest wide use of these tests in China, an upper middle-income country.

Recommendations and suggestions (Table 1).

1) For hospitalized COVID-19 patients in LMICs we suggest $D$-dimer measurement, if available and affordable, at the time of admission for risk stratification, or when clinical suspicion for VTE is high (weak recommendation and low quality of evidence).

2) For hospitalized COVID-19 patients in LMICs, based on current available evidence, we make no recommendation on the use of serial $D$-dimer monitoring for the initiation of therapeutic anticoagulation.

3) For hospitalized COVID-19 patients in LMICs receiving intravenous therapeutic UFH, we recommend serial monitoring of partial thromboplastin time or anti-factor Xa level, based on local laboratory capabilities (strong recommendation and high quality of evidence).

4) For hospitalized COVID-19 patients in LMICs receiving $\mathrm{LMWH}$, we suggest against serial monitoring of anti-factor Xa level (weak recommendation and low quality of evidence).

5) For hospitalized COVID-19 patients in LMICs, we suggest serial monitoring of platelet in patients receiving therapeutic anticoagulation for VTE, to assess risk of bleeding or development of HIT (weak recommendation and low quality of evidence).

Received October 6, 2020. Accepted for publication December 23, 2020.

Published online January 11, 2021.

Note: The Appendix can be found at www.ajtmh.org.

Financial support: Open access fees for this article and all others in this issue were supported by the Wellcome Trust of Great Britain.

Authors' addresses: Hanan Y. Ahmed and Tewodros Haile, Division of Pulmonary and Critical Care Medicine, College of Health Sciences, Addis Ababa University, Addis Ababa, Ethiopia, E-mails: hananyusufahmed@gmail.com and drtewodroshaile@gmail.com. Alfred Papali, Division of Pulmonary and Critical Care Medicine, Atrium Health, Charlotte, NC, E-mail: alfred.papali@atriumhealth.org. Gentle S. Shrestha, Department of Anaesthesiology, Tribhuvan University Teaching Hospital, Kathmandu, Nepal, E-mail: gentlesunder@ hotmail.com. Marcus J. Schultz, Department of Intensive Care, Amsterdam University Medical Centers, Location 'Academic Medical Center,' Amsterdam, The Netherlands, Department of Clinical Tropical Medicine, Mahidol University, Bangkok, Thailand, and MahidolOxford Tropical Medicine Research Unit (MORU), Mahidol University, Bangkok, Thailand, E-mail: marcus.j.schultz@gmail.com. Ganbold Lundeg, Critical Care and Anaesthesia Department, Mongolian National University of Medical Sciences, Ulan Bator, Mongolia, E-mail: ganbold@mnums.edu.mn. Kevan M. Akrami, Faculdade de Medicina, Universidade Federal da Bahia, Salvador, Brazil, and Divisions of Infectious Disease and Critical Care Medicine, University of California San Diego, San Diego, CA, E-mail: kakrami@ucsd.edu.

This is an open-access article distributed under the terms of the Creative Commons Attribution (CC-BY) License, which permits unrestricted use, distribution, and reproduction in any medium, provided the original author and source are credited.

\section{GROUP MEMBERS OF THE "COVID-LMIC TASK FORCE"}

Heads: Alfred Papali (Atrium Health, Charlotte, NC) and Marcus Schultz (Mahidol University, Bangkok, Thailand; University of Oxford, Oxford, United Kingdom; Amsterdam University Medical Centers, location "AMC," Amsterdam, The Netherlands).

Advisors: Neill K. J. Adhikari (Interdepartmental Division of Critical Care Medicine, University of Toronto, Toronto, Canada), Emilie J. Calvello 
Hynes (University of Colorado School of Medicine, Department of Emergency Medicine, Denver, CO), Martin Dünser (Kepler University Hospital and Johannes Kepler University Linz, Linz, Austria), and Mervyn Mer (University of the Witwatersrand, Johannesburg, South Africa).

Subgroup members, in alphabetical order: Andrew Achilleos (Sunnybrook Health Sciences Centre, Toronto, Canada), Hanan Y. Ahmed (Department of Internal Medicine, College of Health Sciences, Addis Ababa University, Addis Ababa, Ethiopia), Kevan M. Akrami (Universidade Federal da Bahia, Salvador, Brazil; University of California San Diego, San Diego, CA), Lia M. Barros (University of Washington, Seattle, WA), B. Jason Brotherton (AIC Kijabe Hospital, Kijabe, Kenya), Sopheakmoniroth Chea (Calmette Hospital, Phnom Penh, Cambodia), William Checkley (The Johns Hopkins University School of Medicine and The Johns Hopkins University Center for Global Health, Baltimore, MD), Elif Cizmeci (Interdepartmental Division of Critical Care Medicine, Sunnybrook Health Sciences Centre, University of Toronto, Toronto, Canada), Natalie Cobb (University of Washington, Seattle, WA), Finot Debebe (Department of Emergency Medicine and Critical Care, Addis Ababa University School of Medicine, Addis Ababa, Ethiopia), Juliana C Ferreira (University of São Paulo, São Paulo, Brazil), Gabriela Galli (University of Pittsburgh School of Medicine, Pittsburgh, PA), Tewodros Haile (Department of Internal Medicine, College of Health Sciences, Addis Ababa University), Bhakti Hansoti (Department of Emergency Medicine, Johns Hopkins University School of Medicine, Baltimore, MD), Madiha Hashmi (Ziauddin University, Karachi, Pakistan), Rebecca Inglis (LaoOxford-Mahosot Hospital-Wellcome Trust Research Unit (LOMWRU), Mahosot Hospital, Vientiane, Lao People's Democratic Republic and Department of Intensive Care, Oxford University Hospital Trust, Oxford, United Kingdom), Burton W. Lee (Critical Care Department, NIH, Bethesda, MD; Division of Pulmonary, Allergy and Critical Care, University of Pittsburgh School of Medicine, Pittsburgh, PA), Faith Lelei (AIC Kijabe Hospital, Kijabe, Kenya), Ganbold Lundeg (Mongolian National University of Medical Sciences, Ulan Bator, Mongolia), David Misango (The Aga Khan University, Nairobi, Kenya), Ary Serpa Neto (Department of Critical Care Medicine, Hospital Israelita Albert Einstein, São Paulo, Brazil; Australian and New Zealand Intensive Care Research Centre (ANZIC-RC), Monash University, Melbourne, Australia; Department of Intensive Care, Amsterdam University Medical Centers, location "AMC," University of Amsterdam, Amsterdam, The Netherlands), Alfred Papali (Atrium Health, Charlotte, NC), Casey Park (Interdepartmental Division of Critical Care Medicine, Sunnybrook Health Sciences Centre, University of Toronto, Toronto, Canada), Rajyabardhan Pattnaik (Ispat General Hospital, Rourkela, India), Jennifer L. Pigoga (Division of Emergency Medicine, University of South Africa, Cape Town, South Africa), Luigi Pisani (Department of Anesthesia and Intensive Care, Miulli Regional Hospital, Acquaviva delle Fonti, Italy; Doctors with Africa - CUAMM, Padova, Italy; Mahidol-Oxford Tropical Medicine Research Unit (MORU), Mahidol University, Bangkok, Thailand), Elisabeth D. Riviello (Division of Pulmonary, Critical Care, and Sleep Medicine, Beth Israel Deaconess Medical Center and Harvard Medical School, Boston, MA), Kristina E. Rudd (University of Pittsburgh School of Medicine, Pittsburgh, PA), Marcus Schultz (Mahidol University, Bangkok, Thailand; University of Oxford, Oxford, United Kingdom; Amsterdam University Medical Centers, location "AMC," Amsterdam, The Netherlands), Varun U. Shetty (University of Pittsburgh Medical Center, Pittsburgh, PA), Gentle S. Shrestha (Tribhuvan University Teaching Hospital, Kathmandu, Nepal), Chaisith Sivakom (Mahidol University, Salaya, Thailand), Shaurya Taran (Interdepartmental Division of Critical Care Medicine, Sunnybrook Health Sciences Centre, University of Toronto, Toronto, Canada), and T. Eoin West (University of Washington, Seattle, WA)

Group members of the subgroup "Anticoagulation": Hanan Y. Ahmed (Department of Internal Medicine, College of Health Sciences, Addis Ababa University, Addis Ababa, Ethiopia), Alfred Papali (Atrium Health, Charlotte, NC), Tewodros Haile (Department of Internal Medicine, College of Health Sciences, Addis Ababa University), Gentle S. Shrestha (Tribhuvan University Teaching Hospital, Kathmandu, Nepal), Marcus Schultz (Mahidol University, Bangkok, Thailand; University of Oxford, Oxford, United Kingdom; Amsterdam University Medical Centers, location "AMC," Amsterdam, The Netherlands), Ganbold Lundeg (Mongolian National University of Medical Sciences, Ulan Bator, Mongolia), and
Kevan M. Akrami (Universidade Federal da Bahia, Salvador, Brazil; University of California San Diego, San Diego, CA).

\section{REFERENCES}

1. Lillicrap D, 2020. Disseminated intravascular coagulation in patients with 2019-nCoV pneumonia. J Thromb Haemost 18: 786-787.

2. Kahn SR et al., 2012. Prevention of VTE in nonsurgical patients: antithrombotic therapy and prevention of thrombosis, 9th ed: American college of chest physicians evidence-based clinical practice guidelines. Chest 141 (Supp/ 2): e195S-e226S.

3. Lodigiani C et al.; Humanitas COVID-19 Task Force, 2020. Venous and arterial thromboembolic complications in COVID-19 patients admitted to an academic hospital in Milan, Italy. Thromb Res 191: 9-14.

4. World Health Organization, 2020. Clinical Management of COVID19: Interim Guidance. Geneva, Switzerland: WHO. Available at: WHO/2019-nCoV/clinical/2020.5.

5. COVID-19 Treatment Guidelines Panel, 2020. Coronavirus Disease 2019 (COVID-19) Treatment Guidelines. National Institutes of Health. Available at: https://www.covid19treatmentguidelines.nih.gov/. Accessed September 24, 2020.

6. Thachil J, Tang N, Gando S, Falanga A, Cattaneo M, Levi M, Clark $\mathrm{C}$, Iba T, 2020. ISTH interim guidance on recognition and management of coagulopathy in COVID-19. J Thromb Haemost 18: 1023-1026.

7. American Society of Hematology, 2020. COVID-19 and VTE/ anticoagulation: Frequently Asked Questions. Available at:https:// www.hematology.org/covid-19/covid-19-and-vte-anticoagulation. Accessed May 22, 2020.

8. Moores LK et al., 2020. Prevention, diagnosis and treatment of venous thromboembolism in patients with COVID-19: CHEST guideline and expert panel report. Chest 158: 1143-1163.

9. Klok FA et al., 2020. Incidence of thrombotic complications in critically ill ICU patients with COVID-19. Thromb Res 191: 145-147.

10. Heit JA, Silverstein MD, Mohr DN, Petterson TM, O'Fallon WM, Melton LJ, 3rd, 1999. Predictors of survival after deep vein thrombosis and pulmonary embolism: a population-based, cohort study. Arch Internal Med 159: 445-453.

11. Tang N, Li D, Wang X, Sun Z, 2020. Abnormal coagulation parameters are associated with poor prognosis in patients with novel coronavirus pneumonia. J Thromb Haemost 18: 844-847.

12. Yao XH et al., 2020. A pathological report of three COVID-19 cases by minimal invasive autopsies. Zhonghua Bing Li Xue Za Zhi 49: 411-417.

13. Bikdeli B et al., 2020. COVID-19 and thrombotic or thromboembolic disease: implications for prevention, antithrombotic therapy, and follow-up. J Am Coll Cardiol 75: 2950-2973.

14. Wang $D$ et al., 2020. Clinical characteristics of 138 hospitalized patients with 2019 novel coronavirus-infected pneumonia in Wuhan, China. JAMA 323: 1061-1069.

15. Poterucha TJ, Libby P, Goldhaber SZ, 2017. More than an anticoagulant: do heparins have direct anti-inflammatory effects? Thromb Haemost 117: 437-444.

16. Alikhan R, Bedenis R, Cohen AT, 2014. Heparin for the prevention of venous thromboembolism in acutely ill medical patients (excluding stroke and myocardial infarction). Cochrane Database Syst Rev 5: CD003747.

17. Ho KM, Tan JA, 2013. Stratified meta-analysis of intermittent pneumatic compression of the lower limbs to prevent venous thromboembolism in hospitalized patients. Circulation 128: 1003-1020.

18. Arabi YM et al., 2019. Adjunctive intermittent pneumatic compression for venous thromboprophylaxis. New Engl J Med 380: 1305-1315.

19. Tapson VF et al., 2007. Venous thromboembolism prophylaxis in acutely ill hospitalized medical patients: findings from the international medical prevention registry on venous thromboembolism. Chest 132: 936-945.

20. Chow JH et al., 2020. Aspirin use is associated with decreased mechanical ventilation, ICU admission, and in-hospital 
mortality in hospitalized patients with COVID-19. Anesth Analg (Epub ahead of print). Available at: https://doi.org/10.1213/ ANE.0000000000005292. Accessed November 20, 2020.

21. British Thoracic Society, 2020. BTS Guidance on Venous Thromboembolic Disease in Patients with COVID-19. London, United Kingdom: British Thoracic Society. Available at: http:// brit-thoracic.org.uk/document-library/quality-improvement/ covid-19/bts-guidance-on-venous-thromboembolic-diseasein-patients-with-covid-19/. Accessed May 22, 2020.

22. Maatman TK et al., 2020. Routine venous thromboembolism prophylaxis may be inadequate in the hypercoagulable state of severe coronavirus disease 2019. Crit Care Med 48: e783-e790.

23. Helms $\mathrm{J}$ et al.; CRICS TRIGGERSEP Group (Clinical Research in Intensive Care and Sepsis Trial Group for Global Evaluation and Research in Sepsis), 2020. High risk of thrombosis in patients with severe SARS-CoV-2 infection: a multicenter prospective cohort study. Intensive Care Medicine 46: 1089-109811.

24. Cui S, Chen S, Li X, Liu S, Wang F, 2020. Prevalence of venous thromboembolism in patients with severe novel coronavirus pneumonia. J Thromb Haemost 18: 1421-1424.

25. Llitjos JF, Leclerc M, Chochois C, Monsallier JM, Ramakers M, Auvray M, Merouani K, 2020. High incidence of venous thromboembolic events in anticoagulated severe COVID-19 patients. J Thromb Haemost 18: 1743-1746.

26. Taccone FS et al., 2020. Higher intensity thromboprophylaxis regimens and pulmonary embolism in critically ill coronavirus disease 2019 patients. Crit Care Med 48: e1087-e1090.

27. Jonmarker $S$ et al., 2020. Dosing of thromboprophylaxis and mortality in critically ill COVID-19 patients. Crit Care 24: 653.

28. Atallah B, Sadik ZG, Salem N, El Nekidy WS, Almahmeed W, Park WM, Cherfan A, Hamed F, Mallat J, 2020. The impact of protocol-based high-intensity pharmacological thromboprophylaxis on thrombotic events in critically ill COVID-19 patients. Anaesthesia (Epub ahead of print). Available at: https://doi.org/ 10.1111/anae.15300.

29. Hsu A, Liu Y, Zayac AS, Olszewski AJ, Reagan JL, 2020. Intensity of anticoagulation and survival in patients hospitalized with COVID-19 pneumonia. Thromb Res 196: 375-378.

30. Longhitano $Y$ et al., 2020. Venous thrombo-embolism in hospitalized SARS-CoV-2 patients treated with three different anticoagulation protocols: prospective observational study. Biology 9: 310.

31. Paranjpe I et al., 2020. Association of treatment dose anticoagulation with in-hospital survival among hospitalized patients with COVID-19. J Am Coll Cardiol 76: 122-124.

32. Tremblay $D$ et al., 2020. Impact of anticoagulation prior to COVID19 infection: a propensity score-matched cohort study. Blood 136: $144-147$.

33. Lynn L, Reyes JA, Hawkins K, Panda A, Linville L, Aldhahri W, Kango G, Shah S, Ayanian S, Teufel K, 2020. The effect of anticoagulation on clinical outcomes in novel coronavirus (COVID-19) pneumonia in a U.S. cohort. Thromb Res 197: 65-68.

34. Nadkarni GN et al., 2020. Anticoagulation, bleeding, mortality, and pathology in hospitalized patients with COVID-19. J Am Coll Cardiol 76: 1815-1826.

35. Ionescu $\mathrm{F}$ et al., 2020. Association of anticoagulation dose and survival in hospitalized COVID-19 patients: a retrospective propensity score-weighted analysis. Eur J Haematol. Available at: https://doi.org/10.1111/ejh.13533.

36. Kamel AM, Sobhy M, Magdy N, Sabry N, Farid S, 2020. Anticoagulation outcomes in hospitalized COVID-19 patients: a systematic review and meta-analysis of case-control and cohort studies. Rev Med Virol e2180. Available at: https://doi.org/ 10.1002/rmv.2180.

37. Lu YF, Pan LY, Zhang WW, Cheng F, Hu SS, Zhang X, Jiang HY, 2020. A meta-analysis of the incidence of venous thromboembolic events and impact of anticoagulation on mortality in patients with COVID-19. Int J Infect Dis 100: 34-41.

38. Ferguson J, Volk S, Vondracek T, Flanigan J, Chernaik A, 2020. Empiric therapeutic anticoagulation and mortality in critically ill patients with respiratory failure from SARS-CoV-2: a retrospective cohort study. J Clin Pharmacol 60: 1411-1415.
39. Billett $\mathrm{HH}$ et al., 2020. Anticoagulation in COVID-19: effect of enoxaparin, heparin, and apixaban on mortality. Thromb Haemost 120: 1691-1699.

40. Lynna L, Reyes JA, Hawkins K, Panda A, Linville L, Aldhahri W, Kango G, Shah S, Ayanian S, Teufel K, 2020. The effect of anticoagulation on clinical outcomes in novel coronavirus (COVID19) pneumonia in a U.S. cohort. Thromb Res 197: 65-68.

41. Southern African Society of Thrombosis and Haemostasis, 2004. Guideline for prophylactic anticoagulation. S Afr Med 94 (8Pt2): 691-695.

42. Anderson FA, Jr., Wheeler HB, Goldberg RJ, Hosmer DW, Forcier A, Patwardhan NA, 1994. Changing clinical practice. Prospective study of the impact of continuing medical education and quality assurance programs on use of prophylaxis for venous thromboembolism. Arch Intern Med 154: 669-677.

43. Stinnett JM, Pendleton R, Skordos L, Wheeler M, Rodgers GM, 2005. Venous thromboembolism prophylaxis in medically ill patients and the development of strategies to improve prophylaxis rates. Am J Hematol 78: 167-172.

44. Kucher N, Koo S, Quiroz R, Cooper JM, Paterno MD, Soukonnikov B, Goldhaber SZ, 2005. Electronic alerts to prevent venous thromboembolism among hospitalized patients. N Engl J Med 352: 969-977.

45. Cohen AT et al., 2008. Venous thromboembolism risk and prophylaxis in the acute hospital care setting (ENDORSE study): a multinational cross-sectional study. Lancet 371: 387-394.

46. Manoucheri R, Fallahi MJ, 2015. Adherence to venous thromboprophylaxis guidelines for medical and surgical inpatients of teaching hospitals, Shiraz-Iran. Tanaffos 14: 17-26.

47. Bikdeli B, Sharif-Kashani B, Raeissi S, Ehteshami-Afshar S, Behzadnia N, Masjedi MR, 2011. Chest physicians' knowledge of appropriate thromboprophylaxis: insights from the PROMOTE study. Blood Coagul Fibrinolysis 22: 667-672.

48. Ma KA, Cohen E, Kahn SR, 2014. Venous thromboembolism in hospitalized patients: an updated analysis of missed opportunities for thromboprophylaxis at a university-affiliated tertiary care center. Vasc Medicine (London, England) 19: 385-391.

49. Ageno W, Squizzato A, Ambrosini F, Dentali F, Marchesi C, Mera V, Steidl L, Veno A, 2002. Thrombosis prophylaxis in medical patients: a retrospective review of clinical practice patterns. Haematologica 87: 746-750.

50. Panda BK, Kumar V, Ketkar MN, 2017. Assessment of risk and prophylaxis for venous thromboembolism and adherence to the American college of chest physicians recommendations during early days of hospital stay at a tertiary care teaching hospital in a developing country. Indian J Vasc Endovasc Surg 4: 107-111.

51. Lee AD, Stephen E, Agarwal S, Premkumar P, 2009. Venous thrombo-embolism in India. Eur $J$ Vasc Endovasc Surg 37: 482-485.

52. Rosovsky RP et al., 2020. Anticoagulation practice patterns in COVID-19: a global survey. Res Pract Thromb Haemost 4: 969-983.

53. Shah A et al., 2020. Thrombotic and haemorrhagic complications in critically ill patients with COVID-19: a multicentre observational study. Crit Care 24: 561.

54. Musoke $\mathrm{N}$ et al., 2020. Anticoagulation and bleeding risk in patients with COVID-19. Thromb Res 196: 227-230.

55. Kvernland $A$ et al., 2020. Anticoagulation use and hemorrhagic stroke in SARS-CoV-2 patients treated at a New York healthcare system. Neurocrit Care (Epub ahead of print). Available at: https:// doi.org/10.1007/s12028-020-01077-0.

56. Palumbo D, Guazzarotti G, De Cobelli F, 2020. Spontaneous major hemorrhage in COVID-19 patients: another brick in the wall of SARS-CoV-2-associated coagulation disorders? J Vasc Interv Radiol 31: 1494-1496.

57. WHO, 2019. World Health Organization Model List of Essential Medicines, 21st List. Geneva, Switzerland: World Health Organization.

58. Fuzinatto F, Waldemar FS, Wajner A, Elias CA, Fernandez JF, Hopf JL, Barreto SS, 2013. A clinical decision support system for venous thromboembolism prophylaxis at a general hospital in a middle-income country. J Bras Pneumol 39: 138-146. 
59. Ceresetto JM, 2016. Venous thromboembolism in Latin America: a review and guide to diagnosis and treatment for primary care. Clinics (Sao Paulo, Brazil) 71: 36-46.

60. Pineo G, Lin J, Stern L, Subrahmanian T, Annemans L, 2012. Economic impact of enoxaparin versus unfractionated heparin for venous thromboembolism prophylaxis in patients with acute ischemic stroke: a hospital perspective of the PREVAIL trial. J Hosp Med 7: 176-182.

61. Deitelzweig SB, Becker R, Lin J, Benner J, 2008. Comparison of the two-year outcomes and costs of prophylaxis in medical patients at risk of venous thromboembolism. Thromb Haemost 100: 810-820.

62. FlaczykA, Rosovsky RP, Reed CT, Bankhead-Kendall BK, Bittner EA, Chang MG, 2020. Comparison of published guidelines for management of coagulopathy and thrombosis in critically ill patients with COVID 19: implications for clinical practice and future investigations. Crit Care 24: 559.

63. Mummery RS, Rider CC,2000. Characterization of the heparinbinding properties of IL-6. J Immunol 165, 5671-5679.

64. Zhou F et al., 2020. Clinical course and risk factors for mortality of adult inpatients with COVID-19 in Wuhan, China: a retrospective cohort study. Lancet 395: 1054-1062.

65. Kartsios C, Lokare A, Osman H, Perrin D, Razaq S, Ayub N, Daddar B, Fair S,2020. Diagnosis, management, and outcomes of venous thromboembolism in COVID-19 positive patients: a role for direct anticoagulants?. J Thromb Thrombolysis (Epub ahead of print). Available at: https://doi.org/10.1007/s11239020-02257-7.

66. Testa $S$ et al., 2020. Direct oral anticoagulant plasma levels' striking increase in severe COVID-19 respiratory syndrome patients treated with antiviral agents: the Cremona experience. J Thromb Haemost 18: 1320-1323.

67. Schiavone $\mathrm{M}$ et al., Oral anticoagulation and clinical outcomes in COVID-19: an Italian multicenter experience. Int J Cardiol 323: 276-280.

68. Alarcon-Calderon A, Celli D, Plate T, Galo J, Alvarez R, 2020. Massive pulmonary embolism treated with thrombolysis in COVID-19: a case series. Chest 158: A2126-A2127.

69. Hirsh J, Warkentin TE, Shaughnessy SG, Anand SS, Halperin JL, Raschke R, Granger C, Ohman EM, Dalen JE, 2001. Heparin and low-molecular-weight heparin: mechanisms of action, pharmacokinetics, dosing, monitoring, efficacy, and safety. Chest 119 (Suppl 1): 64S-94S.

70. Krishnaswamy A, Lincoff AM, Cannon CP, 2010. The use and limitations of unfractionated heparin. Crit Pathways Cardiol 9: 35-40.

71. Holzheimer RG, 2004. Low-molecular-weight heparin (LMWH) in the treatment of thrombosis. Eur J Med Res 9: 225-239.

72. Argenta C, Ferreira MA, Sander GB, Moreira LB, 2011. Short-term therapy with enoxaparin or unfractionated heparin for venous thromboembolism in hospitalized patients: utilization study and cost-minimization analysis. Value Health 14 (5 Suppl 1): S89-S92.

73. Stambler BS, Ngunga LM, 2015. Atrial fibrillation in sub-Saharan Africa: epidemiology, unmet needs, and treatment options. Int $J$ General Med 8: 231-242.

74. Goldstein LN, Wu MT, 2018. A one year audit of patients with venous thromboembolism presenting to a tertiary hospital in Johannesburg, South Africa. Afr J Emerg Med 8: 12-15.

75. Wang T, Chen R, Liu C, Liang W, Guan W, Tang R, Tang C, Zhang $\mathrm{N}$, Zhong N, Li S, 2020. Attention should be paid to venous thromboembolism prophylaxis in the management of COVID19. Lancet Haematol 7: e362-e363.

76. Zhang J, Zhou L, Yang Y, Peng W, Wang W, Chen X, 2020. Therapeutic and triage strategies for 2019 novel coronavirus disease in fever clinics. Lancet Respir Med 8: e11-e12.

77. Williamson DR et al.; PROTECT collaborators, the Canadian Critical Care Trials Group, and the Australian and New Zealand Intensive Care Society Clinical Trials Group, 2013. Thrombocytopenia in critically ill patients receiving thromboprophylaxis: frequency, risk factors, and outcomes. Chest 144: 1207-1215.

78. Lippi G, Plebani M, Henry BM, 2020. Thrombocytopenia is associated with severe coronavirus disease 2019 (COVID-19) infections: a meta-analysis. Clin Chim Acta 506: 145-148.
79. Artifoni M, Danic G, Gautier G, Gicquel P, Boutoille D, Raffi F, Néel $A$, Lecomte R, 2020. Systematic assessment of venous thromboembolism in COVID-19 patients receiving thromboprophylaxis: incidence and role of D-dimer as predictive factors. J Thromb Thrombolysis 50: 211-216.

80. Oudkerk M, Büller HR, Kuijpers D, van Es N, Oudkerk SF, McLoud TC, Gommers D, van Dissel J, Ten Cate H, van Beek EJ, 2020. Diagnosis, prevention, and treatment of thromboembolic complications in COVID-19: report of the national institute for public health of The Netherlands. Radiology 297: E216-E222.

81. Creel-Bulos C, Liu M, Auld SC, Gaddh M, Kempton CL, Sharifpour M, Sniecinski RM, Maier CL, Nahab FB, Rangaraju S, 2020. Trends and diagnostic value of D-dimer levels in patients hospitalized with coronavirus disease 2019. Medicine (Baltimore) 99: e23186.

82. Ooi MWX, Rajai A, Patel R, Gerova N, Godhamgaonkar V, Liong SY, 2020. Pulmonary thromboembolic disease in COVID-19 patients on CT pulmonary angiography - prevalence, pattern of disease and relationship to D-dimer. Eur J Radiol 132: 109336

83. Mouhat B et al., 2020. Elevated D-dimers and lack of anticoagulation predict PE in severe COVID-19 patients. Eur Respir J 56: 2001811

84. Smythe MA, Priziola J, Dobesh PP, Wirth D, Cuker A, Wittkowsky AK, 2016. Guidance for the practical management of the heparin anticoagulants in the treatment of venous thromboembolism. J Thromb Thrombolysis 41: 165-186.

85. Anakwue R, Nwagha T, Ukpabi OJ, Obeka N, Onwubuya E, Onwuchekwa U, Azubuike B, Okoye I, 2017. Clinicians-related determinants of anticoagulation therapy and prophylaxis in Nigeria. Ann Afr Med 16: 164-169.

86. Nwagha TU, Omunakwe HE, 2016. Understanding the RECORDS 3 trial and its impact on anticiagulation practice in resource poor countries. Niger J Clin Pract 19: 695-699.

\section{DEVELOPMENT OF RECOMMENDATIONS AND SUGGESTIONS}

Selection of task force members. The selection of the group members was based on interest in specific aspects of COVID-19 and direct experience in low- and middle-income countries (LMICs). Alfred Papali and Marcus Schultz contacted potential team members through email and in person early in the COVID-19 pandemic and created 10 subgroups assigned to separate areas in COVID-19 management: "triage," "safety," "organization," "diagnostics," "acute respiratory failure," "acute kidney injury," "coagulopathy," "therapeutics," "shock," and "support after initial care." In total, there were 38 Task Force members representing five medical specialties or disciplines (emergency medicine, intensive care, infectious diseases, internal medicine, and critical care nursing) from five of six WHO geographic regions. The task force consisted of 16 full-time LMIC members, 16 full-time HIC members-all with direct LMIC experience-and six members with joint LMIC/HIC appointments.

TABLE A1

Quality of evidence

\begin{tabular}{lll}
\hline A & Randomized clinical trials & High \\
B & $\begin{array}{l}\text { Downgraded randomized clinical trial(s) or } \\
\text { upgraded observational studies }\end{array}$ & Moderate \\
C & $\begin{array}{l}\text { Observational studies } \\
\text { Downgraded observational studies or } \\
\text { expert opinions }\end{array}$ & Low \\
Very low \\
\end{tabular}

Factors that may decrease strength of evidence include high likelihood of bias; inconsistency of results, including problems with subgroup analyses; indirectness of evidence (other population, intervention, control, outcomes, and comparison), imprecision of findings, and likelihood of reporting bias.

Factors that may increase strength of evidence: large magnitude of effect (direct evidence, relative risk $>2$ with no plausible confounders), very large magnitude of effect with relative risk $>5$ and no threats to validity (by two levels), and dose-response gradient (1). 
TABLE A2

Strong vs. weak recommendations*

\begin{tabular}{|c|c|}
\hline What is Considered & How it affects the recommendation \\
\hline High evidence & The higher the quality of evidence, the more likely a strong recommendation \\
\hline $\begin{array}{l}\text { Certainty about the balance of benefits vs. } \\
\text { harms and burdens }\end{array}$ & $\begin{array}{l}\text { The larger/smaller the difference between the desirable and undesirable consequences and the } \\
\text { certainty around that difference, the more likely a strong/weak recommendation }\end{array}$ \\
\hline Certainty in or similar values & $\begin{array}{l}\text { The more certainty or similarity in values and preferences, the more likely a strong } \\
\text { recommendation }\end{array}$ \\
\hline Resource implications & $\begin{array}{l}\text { The lower/higher the cost of an intervention compared to the alternative the more likely a } \\
\text { strong/weak recommendation }\end{array}$ \\
\hline Availability and feasibility in LMICs & The less available, the more likely a weak recommendation \\
\hline Affordability for LMICs & The less affordable, the more likely a weak recommendation \\
\hline Safety of the intervention in LMICs & The less safe in an LMIC, the more likely a weak recommendation \\
\hline
\end{tabular}

Selection of subgroup members. Alfred Papali, Marcus J. Schultz, Hanan Y. Ahmed, Tewodros Haile, Gentle S. Shrestha Ganbold Lundeg, and Kevan M. Akrami were assigned to this subgroup based on their specific expertise and interest in coagulopathy and anticoagulation for patients with COVID-19.

Meetings. The subgroup worked via electronic-based communications to establish the procedures for the literature review, develop relevant questions, and drafting of tables for evidence analysis. Several electronic-based discussions among the subgroup leaders and members occurred.

The first step was to formulate a set of clearly defined questions regarding safety for patients and HCWs caring for patients with suspected or confirmed COVID-19. An initial list of potential questions was reviewed for content and clarity by the subgroup members and leaders from the other subgroups, and questions were rewritten or eliminated after extensive discussion and according to group consensus. This process resulted in three distinct questions.

In the next step, the "anticoagulation" subgroup assigned one or two members to search the literature for evidence to answer each of the questions. The literature search was performed in a minimum of one general database (i.e., MEDLINE and EMBASE) and the Cochrane Libraries, including article pertaining to COVID-19, SARS, MERS, and other respiratory viruses.

Search techniques. In the next step, the "anticoagulation" subgroup assigned one or two members to search the literature for evidence to answer each of the questions. The literature search followed the same techniques as previously described before. ${ }^{1}$ The literature search was performed in a minimum of one general database (i.e., MEDLINE and EMBASE) and the Cochrane Libraries, including article pertaining to COVID-19,
SARS, MERS, and other respiratory viruses. The terms lowresource setting and LMIC were also added to the search, but in cases where adding these terms resulted in no results, we appraised the literature from HICs and adapted the results to the constraints usually present in LMICs. Furthermore, the subgroup members also searched for unpublished study results and included references suggested by group members, when relevant.

Grading of Recommendations. The subgroup members classified quality of evidence as high, moderate, low, or very low and recommendations as strong or weak. The factors influencing this classification are presented in Table A1.

The subgroup members paid extensive attention to several other factors as used before, but now focusing on LMICs, that is, availability and feasibility in LMICs, and safety matters in LMICs. A strong recommendation was worded as "we recommend" and a weak recommendation as "we suggest." Recommendations could remain "ungraded" (UG), when, in the opinion of the subgroup members, such recommendations were not conducive for the process described above (Table A2).

Reporting. The report was edited for style and form by Alfred Papali or Marcus Schultz, with final approval by subgroup leaders and then by the entire "COVID-LMIC Task Force." A final document was submitted to the "American Journal of Tropical Medicine and Hygiene" for potential publication and made open access.

\section{REFERENCE}

1. Dondorp AM, Dünser MW, Schultz MJ, eds., 2019. Sepsis Management in Resource-Limited Settings. Springer. doi.org/ 10.1007/978-3-030-03143-5. 